\title{
Quantitative and qualitative impact of hospital effluent on dissemination of the integron pool
}

\author{
Thibault Stalder ${ }^{1,2,3,4}$, Olivier Barraud ${ }^{1,2,3}$, Thomas Jové ${ }^{5}$, Magali Casellas ${ }^{4}$, \\ Margaux Gaschet ${ }^{1,2}$, Christophe Dagot ${ }^{4}$ and Marie- Cécile Ploy ${ }^{1,2,3}$ \\ ${ }^{1}$ Inserm U1092, Faculté de Médecine, Limoges, France; ${ }^{2}$ Université de Limoges, UMR-S1092, Limoges, \\ France; ${ }^{3} \mathrm{CHU}$ Limoges, Limoges, France; ${ }^{4}$ Université de Limoges, EA4330 GRESE, ENSIL-Ester Technopole, \\ Limoges, France and ${ }^{5}$ Laboratoire de Génétique et Physiologie Bactérienne, Université Libre de Bruxelles \\ Gosselies, Belgium
}

\begin{abstract}
There is increasing evidence that human activity, and especially the resulting effluent, has a major role in the dissemination of bacterial antibiotic-resistance determinants in the environment. Hospitals are the major antibiotic consumers and thus facilitate the spread of antibiotic resistance. Questions are increasingly being raised about the management of hospital effluents, but their involvement in antibiotic-resistance dissemination has never been assessed. Integrons are a paradigm of genetic transfer between the environmental resistome and both commensal and pathogenic bacteria. In order to assess the impact of hospital activities on antibiotic-resistance dissemination in the environment, we monitored integrons and their gene cassettes in hospital effluents, and their release in the environment. We found that bacterial communities present in a hospital effluent contained a high proportion of integrons. In terms of both their gene cassette diversity and gene cassette arrays, the urban effluent and municipal wastewater treatment plant (WWTP) influent were most similar, whereas the hospital effluent and recirculation sludge exhibited very specific patterns. We found that anthropogenic activities led to the release of abundant integrons and antibiotic-resistance gene cassettes, but we observed no specific impact of hospital activities on the receiving environment. Furthermore, although the WWTP did not reduce the normalized integron copy number, it reduced the diversity of gene cassette arrays contained in the raw wastewater, underlining the effect of the biological treatment on the anthropogenic integron pool arriving at the WWTP.
\end{abstract}

The ISME Journal (2014) 8, 768-777; doi:10.1038/ismej.2013.189; published online 24 October 2013

Subject Category: Microbial population and community ecology

Keywords: antibiotic resistance; effluent; hospital; integron; wastewater treatment plant

\section{Introduction}

Integrons are bacterial genetic elements capable of acquiring and expressing genes embedded within gene cassettes (Stokes and Hall, 1989). The integron functional platform is defined by three key elements: (i) intI, a gene encoding an integrase, (ii) attI, a recombination site and (iii) Pc, a promoter controlling gene cassette expression. Gene cassettes are composed of an open reading frame (ORF) and an attC site. Integrase catalyzes gene cassette insertion and excision through attI $\times$ attC or attC $\times$ att $C$ recombination. These powerful genetic tools enable bacteria to adapt to environmental shifts

Correspondence: M-C Ploy, Inserm U1092, Faculté de Médecine, 2, rue du Docteur Marcland, 87065 Limoges, Cedex, France. E-mail: marie-cecile.ploy@unilim.fr

C Dagot, Université de Limoges, EA4330 GRESE, ENSIL-Ester Technopole, 16 rue Atlantis, 87068 Limoges, Cedex, France.

E-mail: dagot@ensil.unilim.fr

Received 3 May 2013; revised 19 September 2013; accepted 22 September 2013; published online 24 October 2013
(Mazel, 2006). Integrons may be located on the bacterial chromosome, in which case they may contain hundreds of cassettes, mostly encoding unknown functions; or on transposons or plasmids, in which case they contain few gene cassettes, usually encoding determinants of antibiotic resistance. Integrons are major determinants of antibiotic resistance in Gram-negative bacteria (Cambray et al., 2010). More than 130 different gene cassettes conferring resistance to most available antibiotics have been identified so far (Partridge et al., 2009). Different classes of integron have been defined according to the sequence of their IntI protein. Class 1 integrons are the most prevalent (Cambray et al., 2010), especially in multidrug-resistant Gram-negative clinical isolates. Class 2 integrons are less frequent, and few class 3 integrons have been described (Barraud et al., 2013). Many known class 1 integrons, especially those of clinical origin, contain, at their $3^{\prime}$ end, a conserved segment composed of three ORFs, namely qacEA1 (low-level resistance to quaternary ammonium compounds 
(QAC)), sul1 (sulfonamide resistance) and orf5 (unknown function) (Gillings et al., 2008a). These class 1 integrons are referred to here as 'resistance integrons' (RI).

Studies of class 1 integrons have shown that (i) class 1 integrons are ubiquitous in the environment, (ii) anthropogenic pressure has a major role in class 1 integron dissemination and (iii) gene cassettes of unknown function are present in class 1 integrons of environmental origin (for review, see Stokes and Gillings, 2011; Stalder et al., 2012). Class 1 integrons have recently been shown to belong to the shared antibiotic resistome of soil bacteria and human pathogens (Forsberg et al., 2012). The conserved structure of class 1 integrons in the bacterial kingdom could constitute a paradigm linking the environmental antibiotic resistome to human and animal pathogenic bacteria. Ecosystems favoring contacts between commensal/pathogenic and environmental bacteria could facilitate horizontal genetic exchanges and, therefore, the dissemination of antibiotic resistance.

Wastewater treatment plants (WWTPs) are a major interface between urban and rural activities and the natural environment (river, sea and soil). WWTP-treated effluents are a significant source of antibiotic-resistance gene release into the downstream environment (Lapara et al., 2011; Wellington et al., 2013). Because of their high nutrient content and high bacterial density, WWTPs are hotspots for dissemination of mobile genetic elements (Szczepanowski et al., 2009). Studies of integrons in these ecosystems have revealed a variable prevalence of class 1 integron-harboring bacteria (Stalder et al., 2012). However, most previous studies involved bacterial isolation, but culture-dependent methods reveal only about $0.1 \%$ to $15 \%$ of the overall bacterial diversity (Amann et al., 1995). Metagenomic studies have underlined the important role of integrons in environmental antibiotic-resistance dissemination via WWTPs (Zhang et al., 2009a; Moura et al., 2010; Gaze et al., 2011). WWTPs collect effluents from diverse sources, including hospitals, private households and factories. Hospitals are high antibiotic and QAC consumers, and are thus considered likely to facilitate antibiotic-resistance gene dissemination in bacterial communities; in addition, their effluents may represent a major source of integrons of anthropogenic origin in both WWTPs and the natural environment (Stalder et al., 2013).

We followed the distribution of class 1,2 and 3 integrons and analyzed the gene cassette content of class 1 RIs from hospital wastewater to a receiving WWTP and its discharge river, by using a cultureindependent molecular approach. The marked diversity of antibiotic-resistance gene cassettes identified here, including gene cassettes previously found in bacteria from wastewater environments and others only detected in clinical strains, supports the important role of integrons in the dissemination of antibiotic resistance in the environment.

\section{Materials and methods}

Study area and sampling procedure

The study was conducted in 2010 and focused on a 900-bed hospital consuming nearly $1000 \mathrm{~m}^{3}$ of water daily. Hospital effluent samples were collected from the medical sewerage system. Other samples were taken from the influent, effluent and recirculation sludge of the municipal WWTP receiving the hospital effluent, as well as from an urban effluent of the town, which has no significant medical or industrial activities (Supplementary Figure S1). The WWTP handles 285000 population equivalents, and the urban effluent analyzed here receives wastewater from 13360 population equivalents. In order to assess the impact of the WWTP on the receiving river, sampling was carried out $2 \mathrm{~km}$ upstream and $3 \mathrm{~km}$ downstream of the discharge point. Flow-proportionate samples were taken over a 24-h period from all sampling points, except for the river and recirculation sludge, where grab samples were collected. All samples were independent and sampling campaigns were conducted on 3 different days during working weeks and on 3 different days during weekends. Ten liters of effluent were collected at $4{ }^{\circ} \mathrm{C}$ in sterile flasks, and were processed within $1 \mathrm{~h}$. A standard pollution assessment was conducted for each sample (see Supplementary Information and Supplementary Table S1).

\section{DNA extraction}

Fresh water samples were vacuum-filtered through sterile $47-\mathrm{mm}$-diameter membranes with a pore size of $0.45 \mu \mathrm{m}$ (Millipore, Billerica, MA, USA). Total bacterial DNA was extracted by using the PowerWater DNA isolation kit (MoBio Laboratories Inc., Carlsbad, CA, USA). For recirculation sludge samples, $2 \mathrm{ml}$ aliquots were pelleted at $15000 \mathrm{~g}$ for $10 \mathrm{~min}$, and total DNA was extracted using the FastDNA Spin kit for feces and the FastPrep Instrument (MP Biomedicals, Illkirch, France). All steps were performed in triplicate.

\section{Quantitative PCRs}

Class 1, 2 and 3 integrons were detected by using a multiplex quantitative PCR (qPCR) Taqman method as described previously (Barraud et al., 2010). Fragments of the 16S rRNA-encoding gene, the $3^{\prime}$ conserved segment, and the aacA4 and $a a d B$ genes were quantified by using a SYBR green-based method assay (for details, see Supplementary Information and Supplementary Table S2). Assays were done in triplicate with the MX3005P detection system (Stratagene-Agilent Technologies, Santa Clara, CA, USA). In order to avoid inhibitor effects, 
DNA samples were diluted to the point where quantification was unaffected. For accurate quantification, the intI1, intI2, intI3 and $16 \mathrm{~S}$ rRNA-encoding genes were embedded in a single plasmid (see Supplementary Information) in order to construct a full-standard curve, between $10^{3}$ and $10^{8}$ copy numbers, which was used in duplicate in each qPCR run. On the basis of the Ribosomal RNA Database, the average number of $16 \mathrm{~S}$ rRNA-encoding genes per bacterium is currently estimated at 4.1 (Klappenbach et al., 2001). 16S rRNA-encoding gene quantities were thus divided by this value to estimate bacterial cell numbers (Hardwick et al., 2008). Moreover, in order to minimize experimental bias, all quantifications of intI and 16S rRNA-encoding genes were performed during the same qPCR run. The Mann - Whitney test was implemented with StatView 5.0 software (SAS Institute Inc., Cary, NC, USA).

Construction of gene cassette array libraries

For class 1 RIs containing the $3^{\prime}$ conserved segment, we used primers $5^{\prime} \mathrm{CS}$ and $3^{\prime} \mathrm{CS}$ (Levesque et al., 1995) to amplify the variable gene cassette-containing region directly from the samples, and the amplicon mixture was used to create clone libraries (see Supplementary Information). Because $5^{\prime} \mathrm{CS}$ and $3^{\prime} \mathrm{CS}$ primers could target nonspecific sites on metagenomic DNA, clones were controlled by PCR with the internal primer MRG284 (Gillings et al., $2008 \mathrm{~b}$ ) adjacent to the $5^{\prime} \mathrm{CS}$ primer hybridization site, coupled to the $3^{\prime} \mathrm{CS}$ primer (see Supplementary Information). The PCR product includes 97 base pairs (bp) of the attI region ( $5^{\prime}$ conserved segment) and $56 \mathrm{bp}$ of the $3^{\prime}$ conserved segment. If the RI contains no gene cassettes (empty RI), the size of the amplification product is $153 \mathrm{bp}$; when the RI contains gene cassettes, the amplicon size increases according to the number of gene cassettes. For each wastewater sample, at least 140 clones were analyzed (see Table 1), and at least 100 clones with a gene cassette array comprising at least 1 gene cassette were used for sequencing with M13 primers on an ABI 3130xl 16-capillary genetic analyzer (Applied Biosystems, Carlsbad, CA, USA).

\section{Analysis of clone libraries}

Sequences were analyzed with Geneious Pro 4.8.4 software (Biomatters, Auckland, New Zealand). After trimming and assembling the sequences, contig sequences were submitted to the entire GenBank nucleotide collection (nr/nt) using the BLASTn alignment search tool (www.ncbi.nlm.nih.gov/BLAST) to check for homology with previously reported gene cassettes. For sequences without homologies in GenBank, potential attC sites were sought manually. All potential ORFs comprised between two putative attC sites were considered to be potential gene cassettes. The protein predictions were submitted to the entire GenBank protein
Table 1 Proportion (\%) of empty class 1 RIs (no gene cassettes) and class 1 RIs harboring at least 1 gene cassette

\begin{tabular}{|c|c|c|c|c|c|c|}
\hline & $H E$ & $U E$ & $W I$ & $W E$ & $R S$ & Resistance phenotype \\
\hline$n$ clone & 143 & 165 & 229 & 194 & 202 & \\
\hline No GC & 13.1 & 24.5 & 50.4 & 45.4 & 80.8 & \\
\hline$\geqslant 1 \mathrm{GC}$ & 86.9 & 75.5 & 49.6 & 54.6 & 19.2 & \\
\hline$a a c A^{\mathrm{a}}$ & 26.7 & 1.5 & 6.9 & 0.0 & 0.0 & Km, Tm, Gm or Ak, \\
\hline $\operatorname{aad} A^{\mathrm{a}}$ & 14.0 & 59.3 & 58.0 & 44.1 & 15.9 & Sm, Sp \\
\hline$a a d B^{\mathrm{a}}$ & 15.7 & 0.0 & 0.0 & 0.0 & 0.0 & $\mathrm{Gm}, \mathrm{Tm}$ \\
\hline bla $_{O X A}{ }^{\mathrm{a}}$ & 21.5 & 9.6 & 16.0 & 20.6 & 13.6 & $\begin{array}{c}\text { Narrow-spectrum } \\
\beta \text {-lactams }\end{array}$ \\
\hline$b l a_{\mathrm{BEL}-1}$ & 0.0 & 0.7 & 0.8 & 0.0 & 0.0 & ESBL \\
\hline$b l a_{\mathrm{GES}-2}$ & 0.0 & 0.7 & 0.0 & 0.0 & 0.0 & ESBL \\
\hline$d f r^{\mathrm{a}}$ & 5.8 & 8.9 & 4.6 & 3.7 & 0.0 & $\mathrm{Tp}$ \\
\hline ere $A 2$ & 0.6 & 0.0 & 0.0 & 0.7 & 0.0 & Em \\
\hline$q a c^{\mathrm{a}}$ & 2.9 & 3.0 & 8.4 & 3.7 & 0.0 & QAC \\
\hline orf & 1.7 & 3.7 & 1.5 & 3.7 & 0.0 & - \\
\hline $\operatorname{orf} f_{\mathrm{C}-\mathrm{LIM}}$ & 11.0 & 12.6 & 3.8 & 23.5 & 70.5 & - \\
\hline
\end{tabular}

Abbreviations: Ak, amikacin; Em, erythromycin; ESBL, extendedspectrum $\beta$-lactamase; GC, gene cassette; Gm, gentamicin; HE, hospital effluent; Km, kanamycin; QAC, quaternary ammonium compound; RS, recirculation sludge; Sm, streptomycin; Sp, spectinomycin; Tm, tobramycin; Tp, trimethoprim; UE, urban effluent; WE, WWTP effluent; WI, WWTP influent.

$\mathrm{n}$ clone indicates the number of clones analyzed.

${ }^{a}$ Indicates allele.

collection, using BLASTp (www.ncbi.nlm.nih.gov/ BLAST). Alignments of the predicted secondary protein structures were carried out using the Phyre2 webserver (www.sbg.bio.ic.ac.uk/phyre2). The nucleotide sequences of gene cassette arrays including a new gene cassette, or a variant of a known gene cassette, have been deposited in the GenBank data library under accession nos. KF525275 to KF525340.

Analysis of gene cassette array diversity

For all subsequent analyses, a sequence identity threshold of $99 \%$ was used to cluster gene cassettes. Rarefaction analyses (Supplementary Figure S2) were performed for each clone library, using PAST software (PAleontological Statistics v1.60) from http://folk.uio.no/ohammer/past/. Primer6 software (Plymouth Routines In Multivariate Ecological Research, version 6.1.6) was used to calculate diversity indices (Chao 1 richness estimator and Shannon diversity indexes), and to perform the clustering analysis coupled with two-dimensional non-metric multidimensional scaling ordination based on the Bray-Curtis similarity index. The coverage value of the clone libraries was calculated according to Good 1953, as $C=1-\left(n_{1} / \mathrm{N}\right) \times 100$, where $n_{1}$ is the number of clones that occurred only once in the library.

\section{Results}

Integron pool and impact of hospital activities on integron dissemination in a WWTP

Integrons were detected at high concentrations in all raw wastewater and recirculation sludge, the sum of the quantifications for the three integron classes 
reaching $10^{10}-10^{12}$ copies. $1^{-1}$ or copies.g ${ }^{-1}$ of dry matter (Figure 1). Class 1 integrons were significantly more prevalent $(P<0.01)$ than class 2 and 3 integrons. Unexpectedly, class 3 integrons were more abundant $(P<0.01)$ than class 2 integrons both in the wastewater continuum and in environmental samples. In order to normalize the quantity of integrons in the total bacterial community, all intI quantifications were normalized to the molecularly estimated bacterial cell number. Except for class 3, all concentrations and normalized integron copy numbers were the highest $(P<0.01)$ in the hospital effluent (Figure 1).

The qPCR method used here is able to detect integrons from both clinical and environmental settings as previously defined (Gillings et al., 2008a). Specific quantification of class 1 RIs, which contain the $3^{\prime}$ conserved segment, showed that these RIs represented one-half to one-fifth of the entire quantity of class 1 integrons detected, depending on the effluent (Supplementary Figure S3). The variations of normalized copy numbers for the $3^{\prime}$ conserved segment observed between samples were similar to those observed for class 1 integrons.

To assess the impact of hospital effluent on integron dissemination in the WWTP, samples were collected both during the working week and at the weekend. The normalized copy number of class 1 integrons in the hospital effluent was higher $(P<0.01)$ during the working week, whereas no difference was observed in other samples (weekend data not shown). Integron concentrations were also
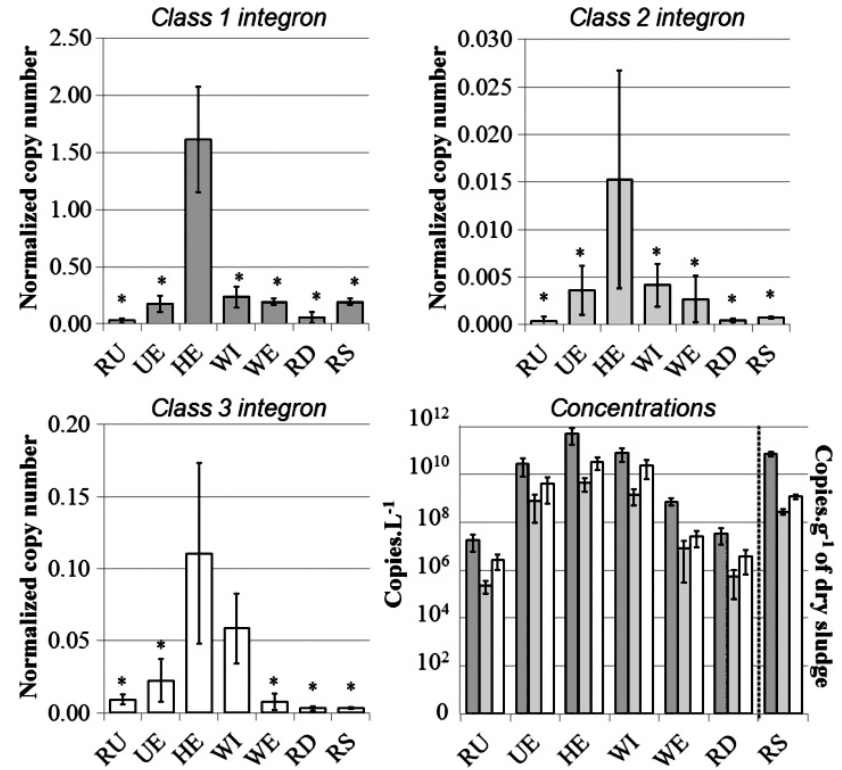

Figure 1 Normalized integron copy number (number of integrons per bacterial cell) and concentrations of integrons in the total bacterial community at the different points sampled during the working week. Class 1 integrons: dark gray bars, class 2 integrons: gray bars and class 3 integrons: white bars. HE, hospital effluent; RS, recirculation sludge; RD, river downstream of the WWTP; RU, river upstream of the WWTP; UE, urban effluent; WE, WWTP effluent; WI, WWTP influent. ${ }^{*} P<0.01$ compared with the hospital effluent. analyzed according to flow data (Supplementary Table S3), and the contribution of hospital effluent was confirmed to be lower $(P=0.03)$ during the weekend. Moreover, although the hospital effluent contributed only $2.2 \%$ to the wastewater flow arriving at the WWTP during the working week, $13.8 \%$ of class 1 integrons originated in the hospital effluent (Supplementary Table S4).

During the working weeks, about $95.4 \%$ of all integrons detected and $91.6 \%$ of all bacteria arriving in the WWTP were eliminated, corresponding to around a 2-log decrease in their concentrations (Figure 1). The remaining fraction was not only found mainly in the recirculation sludge $(4.0 \%$ of intI genes) but also in the WWTP effluent $(0.6 \%)$, corresponding to the release of $2.87 \times 10^{16}$ copies daily into the river. The normalized copy number of class 1 and 2 integrons was unchanged $(P>0.05)$ during transit from the WWTP influent to the WWTP effluent, whereas that of class 3 integrons fell $(P<0.01)$ (Figure 1). The normalized copy number of class 1 integrons in the recirculation sludge was similar to that found in the WWTP influent (Figure 1).

There was no difference $(P>0.05)$ in the quantity of integrons between upstream and downstream river sampling points, probably owing to dilution (nearly 90-fold) of the WWTP effluent in the river. For class 1 RIs, even if we observed a higher normalized copy number in the downstream point, the values were very low in the river (upstream and downstream) (Supplementary Figure S3B).

Gene cassette array and gene cassette characterization To further characterize the impact of hospital activities on integrons and antibiotic-resistance gene cassette dissemination, we focused on the gene cassette content of class 1 RIs. We found that $55.9 \%$ of RIs were empty, especially those in the recirculation sludge (Table 1). Among the clones analyzed, RIs carrying at least one gene cassette were more prevalent in the hospital effluent than in the other effluents.

The majority of the gene cassette arrays identified in this study were absent from nucleotide sequence databases. Some gene cassette arrays not detected in the urban effluent were found in the hospital effluent and also, in lower proportions, in the WWTP influent (Supplementary Table S5).

The gene cassettes aacA (mostly represented by $a a c A 4$ ) and $a a d B$, conferring resistance to aminoglycosides, were mainly found in the hospital effluent (Table 1 and Figure 2). We confirmed, with specific qPCRs, that the aacA4 and $a a d B$ gene cassettes were significantly more abundant $(P<0.01)$ in hospital effluent than in the other wastewater samples (Supplementary Figure S4).

Other gene cassettes were found in the different samples (Table 1 and Figure 2), encoding resistance to streptomycin and spectinomycin $(\operatorname{aad} A)$, trimethoprim (dfr), QACs (qac), $\beta$-lactams (bla) or 


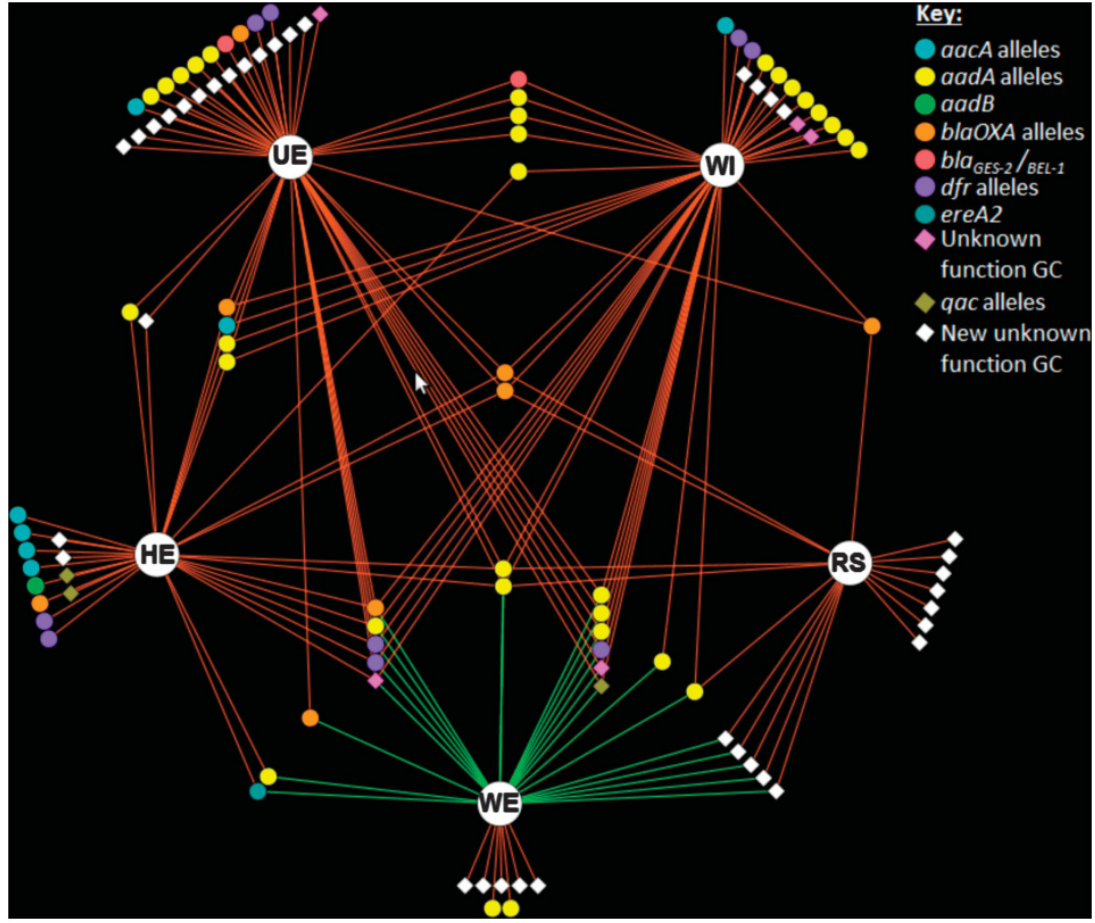

Figure 2 Network based on gene cassette diversity recovered from each site. The sampling sites are represented by large white dots, and each small dot or diamond represents a different gene cassette (based on $\geqslant 99 \%$ nucleic acid identity of clustered gene cassettes). Dots represent gene cassettes related to antibiotic resistance, and diamonds represent gene cassettes with other related functions. The color key attributes each gene cassette to a related function.

erythromycin (ere). Thirty-two different aadA alleles were characterized (Supplementary Table S6), of which 20 were likely new aadA variants (less than $99 \%$ identity with known aadA gene cassettes).

A high diversity $(n=37)$ of new gene cassettes was identified in all samples. In the recirculation sludge, these new gene cassettes represented $70 \%$ of all the gene cassettes recovered. They were predicted to encode proteins of unknown function (orf $f_{\text {C-LIM }}$; Supplementary Table S6 and Figure 2). orf $_{\text {C-LIMg }}$, which was specific to hospital effluent, potentially encodes a protein sharing high protein sequence identity (73\%) with YP_00600849, as well as structural homology $(98 \%)$ with AlbG (CAE52332), the two proteins belonging to the large family of pentapeptide repeat proteins. The pentapeptide repeat protein family also includes Qnr proteins involved in fluoroquinolone resistance. Interestingly, AlbG is known to be involved in resistance to the topoisomerase inhibitor albicidin, which has a mechanism of action analogous to that of fluoroquinolones. Two new gene cassettes, $\operatorname{orf}_{C-L I M 7}$ and $\operatorname{orf}_{C-L I M 33}$, potentially encode proteins related to chromosomal integron (CI)-encoded proteins: $\operatorname{orf}_{C-L I M 7}$ appears to encode a protein sharing $72 \%$ identity with the hypothetical protein Alide2_3696 described in the Alicycliphilus denitrificans K601 CI, whereas orf C-LIM33 $_{\text {would encode a }}$ protein sharing $65 \%$ identity with the hypothetical protein VVMO6_01371 described in the Vibrio vulnificus MO6-24/O (YP_004188596) CI.
The $\mathrm{R}^{\prime \prime}$ and $\mathrm{R}^{\prime}$ sequences specific to the attC sites were identified in the new gene cassettes. Several attC sites shared strong identity with attC sites previously found in CIs of environmental bacteria (Figure 3).

\section{Diversity of gene cassettes}

The urban effluent and WWTP influent represented the most diverse samples in terms of gene cassette arrays and gene cassettes (Supplementary Figure S2 and Supplementary Table S7). However, as the rarefaction curves were unsaturated, the gene cassettes identified here represented only a fraction of the total diversity of the gene cassette arrays at the sampling sites. For the hospital effluent, WWTP effluent and recirculation sludge, the rarefaction curves tended to saturation, and we found that these three sites exhibited the lowest gene cassette array diversity. The Bray-Curtis similarity index was used to analyze qualitatively the similarity between samples in terms of both gene cassette diversity and gene cassette arrays. We found that the urban effluent and WWTP influent were most similar, whereas the hospital effluent and the recirculation sludge exhibited very specific patterns (Figure 4).

\section{Discussion}

The aim of this study was to assess the impact of hospital activities on the dissemination of bacterial 


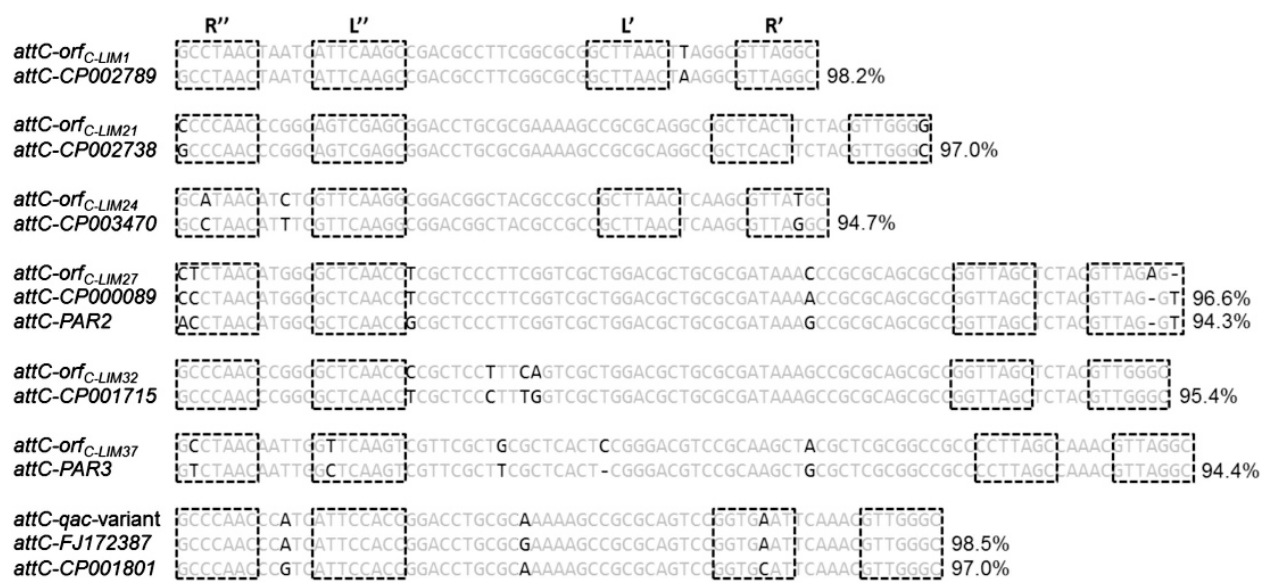

Figure 3 Alignment of $a t t C$ recombination sites of the gene cassettes with known CI-attC sites. The dotted box indicates the inverted repeats (L' and $\mathrm{L}^{\prime \prime}, \mathrm{R}^{\prime}$ and $\mathrm{R}^{\prime \prime}$ ); attC-CP002789: attC site of the CI from X. campestris pv. raphani 756C (CP002789); attC-CP002738: attC site of the CI from M. methanica MC09 (CP002738); attC-CP003470: attC site of the CI from Rhodanobacter sp. 2APBS1 (CP003470); attC-CP000089: attC site of the CI from D. aromatic RCB (CP000089); attC-CP001715: attC site of the CI from Ca. A. phosphatis clade IIA str. UW-1 (CP001715); attC-FJ172387: attC site of a class 1 integron hypothetical protein-encoding gene cassette from an uncultured bacterium (FJ172387); attC-CP001801: attC site of the CI of $H$. neapolitanus C2 (CP001801); attC-PAR2 and attC-PAR3: attC sites of the CI from $P$. alcaligenes ATCC55044 (AY038186); the percentage identity between the attC site of this study and the previously reported attC site is indicated on the right of the alignment.

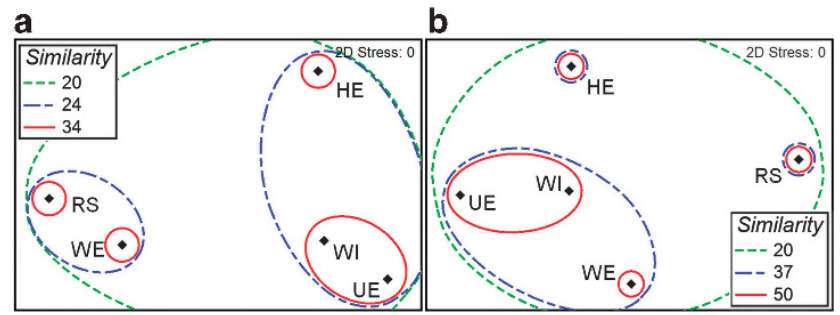

Figure 4 nMDS plot representing, on a two dimensional scale, the projection of the Bray - Curtis similarity index for (a) the gene cassette array and (b) the gene cassette pool. HE, hospital effluent; RS, recirculation sludge; RD, river downstream of the WWTP; RU, river upstream of the WWTP; UE, urban effluent; WE, WWTP effluent; WI, WWTP influent.

RI and antibiotic-resistance gene cassettes. Class 1 integrons were the most frequent integrons in all samples, and their concentrations in the WWTP influent and effluent were similar to those previously reported in such samples (Zhang et al., 2009a, b). The high normalized copy number of class 1 integrons in wastewater samples supports the view that human activities are a major source of class 1 integrons (Wright et al., 2008; Moura et al., 2010; Rosewarne et al., 2010). Moreover, the marked diversity of antibiotic-resistance gene cassettes identified here, including gene cassettes previously found in bacteria from wastewater environments and others only detected in clinical strains (Supplementary Table S6), supports the important role of class 1 integrons in the dissemination of antibiotic resistance.

\section{Hospital effluents: evidence of antibiotic selective} pressure

The hospital effluent contained the highest concentrations and highest normalized copy number of class 1 integrons, half of these integrons having the structure of class 1 RIs. Differences in the relative abundance of class 1 integrons between the working week and the weekend suggest that clinical activities influence the release of class 1 integronharboring bacteria. Indeed, some clinical activities are less intensive during the weekend (outpatient clinics, day hospital). Moreover, the proportion of class 1 RIs with at least one gene cassette was higher in the hospital effluent than in the other samples, which contained a higher proportion of empty RIs. All these results highlight the significant role played by class 1 RIs in antibiotic-resistance dissemination in the hospital setting.

Most of the gene cassettes found in the hospital effluent conferred resistance to antibiotics frequently used in the hospital setting, suggesting that antibiotic selective pressure maintains RI-containing bacteria (Figure 5).

The gene cassettes $a a c A 4$ and $a a d B$ encoding resistance to aminoglycosides (amikacin, gentamicin, tobramycin and netilmicin), which are used almost exclusively in hospitals, were significantly more abundant in the hospital effluent (Table 1; Supplementary Figure S4), suggesting that hospital antibiotic pressure selected bacteria-harboring RIs with aminoglycoside-resistance gene cassettes. This specific hospital antibiotic pressure was confirmed by data from the French Institute for Public Health Surveillance in 2010, showing that the national exposure density to aminoglycosides was 11 times higher in hospitals than in the community (0,0 defined daily doses (DDD) per 1000 patientdays in the community versus 11.4 DDD per 1000 hospitalization-days) (Cavalié, 2012). For the hospital studied here, the aminoglycoside DDD per 1000 hospitalization-days was 9.6.

Hospitals are also large QAC consumers (Kümmerer, 2001), and QACs have been shown to 
be involved in class 1 integron dissemination (Gaze et al., 2005; Gillings et al., 2008b; Gaze et al., 2011). Surprisingly, we detected few qac gene cassettes in the gene cassette arrays in the hospital effluent. However, we focused only on class 1 RIs that contained a QAC-resistance determinant, the $3^{\prime}$ conserved segment harboring the low-level resistance qacEA1 gene variant or its complete version qacE (Stokes and Gillings, 2011).

Urban effluent: a source of gene cassette diversity By comparison with the hospital effluent, the urban effluent contained a lower concentration and a lower normalized copy number (10-fold) of bacteria harboring class 1 integrons. Furthermore, the urban effluent contained a smaller proportion of class 1 RIs harboring at least one gene cassette, although gene cassette diversity was broader than in the hospital effluent.

Surprisingly, the extended-spectrum $\beta$-lactamaseencoding gene cassettes, $b l a_{\mathrm{GES}-2}$ and $b l a_{\mathrm{BEL}-1}$, were found in the urban effluent and not in the hospital effluent (Supplementary Table S5). These two gene cassettes have previously been described only in clinical strains from Belgium and South Africa, respectively (Poirel et al., 2001, 2005). However, $\beta$-lactams that could select such extended-spectrum $\beta$-lactamase gene cassettes are used both in hospitals and in the community. These gene cassettes encode resistance to broad-spectrum $\beta$-lactams, and their dissemination is a public health concern because therapeutic options for these organisms are limited.

\section{The WWTP: a biological reactor for anthropogenic} and environmental integrons

The WWTP influent most closely resembled the urban effluent in terms of both their quantitative and qualitative class 1 integron composition, showing that the urban effluent sampled in this study was representative of the total urban effluents. The observed bacterial elimination (2 log) after passage through the WWTP is typical of activated sludge processes (Łuczkiewicz et al., 2010; Novo and Manaia, 2010). Although more than $90 \%$ of integrons were eliminated, the normalized copy number of class 1 and class 2 integrons was not reduced, and around $10^{16}$ integrons were released each day into environmental water (Figure 5). It has previously been shown that activated sludge processes, which reduce total bacterial loads, do not specifically eliminate bacteria harboring integrons (Ferreira da Silva et al., 2007; Zhang et al., 2009a). Nevertheless, we found that the WWTP reduced the diversity of gene cassette arrays contained in the raw wastewater, underlining the effect of the biological treatment on the anthropogenic integron pool arriving at the WWTP.

In the recirculation sludge, which represents a significant source of integron dissemination into the

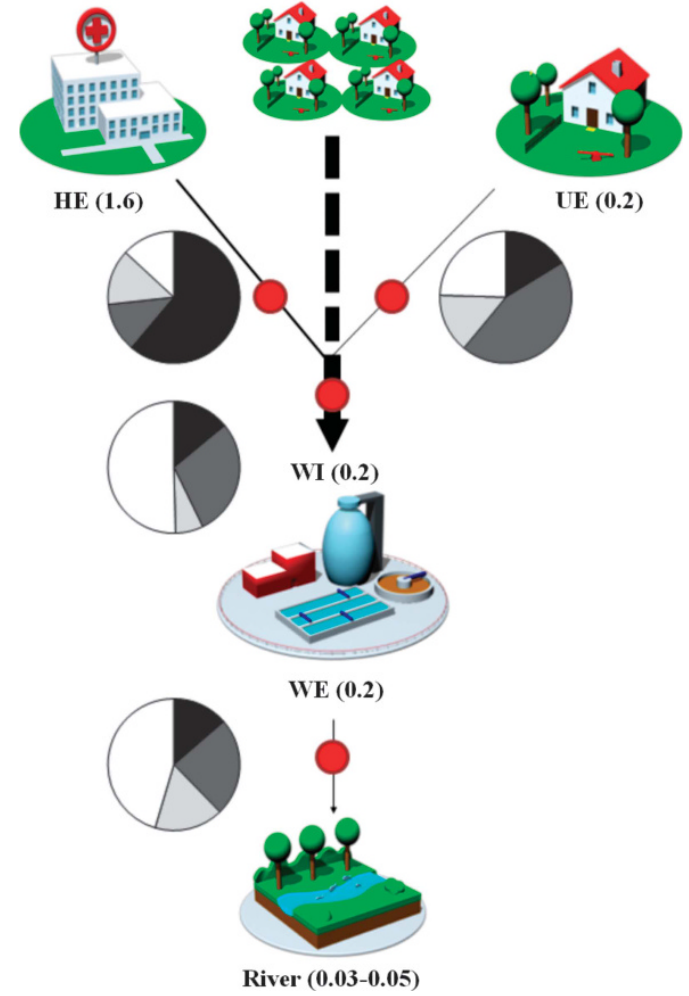

Figure 5 Integron flow, normalized integron copy number (in parentheses) and class 1 RI gene cassette composition along the continuum. Red dots represent the sampling points. Arrows represent the integron flow. The dashed line represents the unsampled urban effluent. The width of the arrows is proportional to the integron flow, considering the flow at the WWTP entry point as $100 \%$; circles represent the proportion of gene cassette types harbored by class 1 RIs at each location: black quarters represent related antibiotic-resistance gene cassettes other than the $\operatorname{aad} A$ gene cassettes; dark gray quarters represent aad $A$ gene cassettes; gray quarters represent other gene cassettes; and white quarters represent the fraction of empty integrons. HE, hospital effluent; RS, recirculation sludge; UE, urban effluent; WE, WWTP effluent; WI, WWTP influent.

environment (sludge is sometimes spread on fields as a fertilizer), most class 1 RIs were empty (Figure 5). Empty class 1 RIs have been reported in QAC-contaminated reed beds, activated sludge and manured soils (Binh et al., 2009; Gaze et al., 2011). These empty RIs do not contain gene cassettes between the $5^{\prime}$ and $3^{\prime}$ conserved segments, but contain qacE $\Delta 1$ or qacE determinants. Thus, a large number of these empty RIs could reflect selective pressure by QACs in the environment (Gaze et al., 2011). Moreover, the bacterial biomass in activated sludge is submitted to stressors (for example, starvation, oxic/anoxic conditions and anthropogenic chemicals released by effluents, including antibiotics). Even if antibiotics concentrations in WWTP are too low to exert direct selective pressure on bacterial communities (Bruchmann et al., 2013; Rizzo et al., 2013), in vitro studies have shown that subinhibitory antibiotic concentrations could impact the behavior of bacterial communities and could influence genetic modifications in bacteria 
(Rodríguez-Rojas et al., 2013), thus potentially leading to enrich the global resistome of the WWTP. We have previously shown that integrase expression is under the control of the SOS response, which is activated by environmental stressors, including antibiotics (Guerin et al., 2009). When expressed, the integrase catalyzes gene cassette insertion and excision. Global sludge stress constitutes an ideal system for activation of the SOS response, leading to continuous IntI expression and, thus, to a high rate of gene cassette rearrangement via excision, increasing the probability of empty RIs.

The normalized copy number of integrons in the discharged river water was similar to that found in previous studies (Hardwick et al., 2008; Gillings et al., 2008b; Wright et al., 2008; Rosewarne et al., 2010), in which bacteria harboring class 1 integrons represented between $1 \%$ and $10 \%$ of total bacteria in various environmental samples. These low values seem to represent the basal integron background occurring naturally in bacterial communities not subjected to anthropogenic activities. Nevertheless, we cannot rule out the occurrence of integrons of anthropogenic origin at this point, as the river goes through a livestock area upstream of the city. Due to the dilution effect, the WWTP effluent does not seem to have a noteworthy impact on the concentration and normalized copy number of integrons in the receiving river. Several studies of the impact of treated wastewater effluents on receiving rivers have shown the introduction of resistance genes or resistance genetic elements in downstream environments (Rosewarne et al., 2010; Lapara et al., 2011; Uyaguari et al., 2011). It would be interesting to further investigate the concentration of integrons and their gene cassette contents in river sediments or biofilms.

\section{Gene cassette diversity and shuffling}

We found a large diversity of gene cassettes at the different sampling sites. For some gene cassettes, such as $\operatorname{aad} A$, numerous alleles were found. When we analyzed the association of the genes with their attC site, we found that most alleles were associated with the attC site of the original gene cassette, suggesting that the variants were derived from the genuine genes. Moreover, we observed that the proportion of gene cassette variants in the hospital effluent was very low but increased along the wastewater flow. Antibiotics might indeed enrich the more efficient adaptive genes (Blázquez et al., 2012; Baquero et al., 2013).

Our analysis of the network of gene cassette diversity in all the sampling sites highlighted the shuffling of gene cassettes between the anthropogenic and environmental ecosystems. Figure 2 shows that the diversity of gene cassettes was higher in urban effluent than in hospital effluent, and that WWTP effluent logically contained a mix of gene cassettes from the different effluents. Some antibiotic resistance gene cassettes were common to the different effluents and the recirculation sludge. In treated effluent, gene cassettes from the recirculation sludge, predicted to encode mainly proteins of unknown function, were mixed with antibiotic-resistance gene cassettes from raw wastewater. This mixing is thought to enhance the genetic diversity and to promote exchanges between bacteria of anthropogenic origin and environmental bacteria indigenous to activated sludge (Baquero et al., 2008; Martínez, 2009). This is underlined by new associations, in WWTP effluent and recirculation sludge, of known antibiotic-resistance gene cassettes with gene cassettes of unknown function in the same array (orf C-LIM 35 -aadA13, $\operatorname{orf}_{\text {C-LIM } 34}-$ bla $_{\text {OXA-35 }}$ and $\operatorname{orf}_{\text {C-LIM } 36}-\operatorname{aadA2}$ ).

Gene cassette uptake and integron shuffling are powerful adaptive mechanisms of host bacteria. Most class 2 integrons have a non-functional integrase and are thus unable to acquire new gene cassettes, limiting their access to the vast diversity of gene cassettes and reducing their role in antibiotic-resistance dissemination. This could explain their low abundance in the environment. The success of class 1 integrons in colonizing a wide diversity of ecosystems (environmental, clinical and veterinary settings) could be linked to the fact that IntI1 recognizes a wide range of attC sites, thus providing access to a broad diversity of gene cassettes (Larouche and Roy, 2011). Furthermore, the evolutionary history of class 1 integrons could explain their abundance in different ecosystems. Indeed, they were associated in the pre-antibiotic era with diverse plasmids or transposons (such as Tn21) that are widespread in environmental bacteria and contain QAC-resistance or mercury-resistance determinants. Exposure to heavy metals and QACs may have exerted selective pressure, enriching bacterial communities with integrons, before the antibiotic era (Gillings et al., 2008b; Rosewarne et al., 2010; Stokes and Gillings, 2011).

\section{Links between CIs and RIS}

Human activities increase the prevalence of integrons in environmental bacterial communities, thereby increasing the metagenomic plasticity of bacterial communities and enhancing their evolution. The gene cassette metagenome of environmental bacteria is a vast and diverse genetic resource that enhances bacterial functional diversity (Koenig et al., 2008; Moura et al., 2010; Elsaied et al., 2011). In class 1 RIs, we identified two new gene cassettes (orf $f_{L I M 7}$ and $\operatorname{orf}_{L I M 33}$ ) encoding putative proteins resembling CI-encoded proteins found in A. denitrificans and $V$. vulnificus. In addition, we found different attC sites of some new gene cassettes from class 1 RIs, which shared identity (>94\%) with CI-attC sites previously described in environmental bacteria (Xanthomonas campestris, Methylomonas methanica, Rhodanobacter spp., 
Candidatus Accumulibacter phosphatis, Halothiobacillus neapolitanus, Pseudomonas alcaligenes and Dechloromonas aromatica). It has been shown in vitro that the integrase of class 1 integrons can recruit gene cassettes from CIs (Rowe-Magnus et al., 2002). We suspect that these gene cassettes detected in RIs could originate from CIs. This is supported by our metagenomic bacterial diversity analysis, based on pyrosequencing of a fragment of the 16S rRNAencoding gene (see Supplementary Information). This showed the presence of bacteria phylogenetically related to $D$. aromatica, $P$. alcaligenes and Xanthomonas spp. in the same samples as those in which orf $f_{C-L I M 1}$, orf $f_{\text {C-LIM27 }}$ and $\operatorname{orf}_{\text {C-LIM37 }}$ gene cassettes were found. This link between the gene cassette pools of CIs and RIs has previously been described (Mazel, 2006; LeRoux et al., 2009; Koenig et al., 2011).

\section{Conclusions}

This study provides a quantitative and qualitative view of the role of both hospital and urban effluent in antibiotic-resistance dissemination, via the integron marker, from their emission to their release into the environment. We found that anthropogenic activities led to the release of abundant integrons and antibiotic-resistance gene cassettes. However, we did not observe a specific impact of hospital activities on the receiving environment. Interestingly, two gene cassettes (aacA4 and $a a d B$ ) were mostly found in the hospital effluent. Further studies in other hospitals are needed to confirm whether they might constitute markers of clinical activity.

\section{Conflict of Interest}

The authors declare no conflict of interest.

\section{Acknowledgements}

This work was supported by the European INTEREG program PILLS, the Limousin region and the CPAM HauteVienne. We thank the city and hospital of Limoges, as well as Didier Mazel and Sandra Da-Re for their critical reading of the manuscript and Armelle Marie-Daragon for providing data on antibiotic consumption. Thibault Stalder was funded by grants from the Limousin region.

\section{References}

Amann RI, Ludwig W, Schleifer KH. (1995). Phylogenetic identification and in situ detection of individual microbial cells without cultivation. Microbiol Rev 59: 143-169.

Baquero F, Martínez JL, Cantón R. (2008). Antibiotics and antibiotic resistance in water environments. Curr Opin Biotechnol 19: 260-265.

Baquero F, Tedim AP, Coque TM. (2013). Antibiotic resistance shaping multi-level population biology of bacteria. Front Microbiol 4: 15.
Barraud O, Baclet MC, Denis F, Ploy M-C. (2010). Quantitative multiplex real-time PCR for detecting class 1, 2 and 3 integrons. J Antimicrob Chemother 65: 1642-1645.

Barraud O, Casellas M, Dagot C, Ploy M-C. (2013). An antibiotic-resistant class 3 integron in an Enterobacter cloacae isolate from hospital effluent. Clin Microbiol Infect 19: E306-E308.

Binh CTT, Heuer H, Kaupenjohann M, Smalla K. (2009). Diverse aadA gene cassettes on class 1 integrons introduced into soil via spread manure. Res Microbiol 160: $427-433$.

Blázquez J, Couce A, Rodríguez-Beltrán J, Rodríguez-Rojas A. (2012). Antimicrobials as promoters of genetic variation. Curr Opin Microbiol 15: 561-569.

Bruchmann J, Kirchen S, Schwartz T. (2013). Sub-inhibitory concentrations of antibiotics and wastewater influencing biofilm formation and gene expression of multi-resistant Pseudomonas aeruginosa wastewater isolates. Environ Sci Pollut Res Int 20: 3539-3549.

Cambray G, Guerout AM, Mazel D. (2010). Integrons. Annu Rev Genet 44: 141-166.

Cavalié P. (2012). Trends in antibiotic use in France in 2000 - 2010. Bull épidémiol hebd 42-43: 480-485.

Elsaied H, Stokes HW, Kitamura K, Kurusu Y, Kamagata Y, Maruyama A. (2011). Marine integrons containing novel integrase genes, attachment sites, attI, and associated gene cassettes in polluted sediments from Suez and Tokyo Bays. ISME J 5: 1162-1177.

Ferreira da Silva M, Vaz-Moreira I, Gonzalez Pajuelo M, Nunes OC, Manaia CM. (2007). Antimicrobial resistance patterns in Enterobacteriaceae isolated from an urban wastewater treatment plant. FEMS Microbiol Ecol 60: 166-176.

Forsberg KJ, Reyes A, Wang B, Selleck EM, Sommer MOA, Dantas G. (2012). The shared antibiotic resistome of soil bacteria and human pathogens. Science 337: 1107-1111.

Gaze WH, Abdouslam N, Hawkey PM, Wellington EMH. (2005). Incidence of class 1 integrons in a quaternary ammonium compound-polluted environment. Antimicrob Agents Chemother 49: 1802-1807.

Gaze WH, Zhang L, Abdouslam NA, Hawkey PM, Calvo-Bado L, Royle J et al. (2011). Impacts of anthropogenic activity on the ecology of class 1 integrons and integron-associated genes in the environment. ISME J 5: 1253-1261.

Gillings MR, Boucher Y, Labbate M, Holmes A, Krishnan S, Holley M et al. (2008a). The evolution of class 1 integrons and the rise of antibiotic resistance. J Bacteriol 190: 5095-5100.

Gillings MR, Xuejun D, Hardwick SA, Holley MP, Stokes HW. (2008b). Gene cassettes encoding resistance to quaternary ammonium compounds: a role in the origin of clinical class 1 integrons? ISME J 3: 209-215.

Guerin E, Cambray G, Sanchez-Alberola N, Campoy S, Erill I, Da Re S et al. (2009). The SOS response controls integron recombination. Science 324: 1034.

Hardwick SA, Stokes HW, Findlay S, Taylor M, Gillings MR. (2008). Quantification of class 1 integron abundance in natural environments using real-time quantitative PCR. FEMS Microbiol Lett 278: 207-212.

Klappenbach JA, Saxman PR, Cole JR, Schmidt TM. (2001). rrndb: the ribosomal RNA operon copy number database. Nucleic Acids Res 29: 181-184. 
Koenig JE, Boucher Y, Charlebois RL, Nesbø C, Zhaxybayeva O, Bapteste E et al. (2008). Integronassociated gene cassettes in Halifax Harbour: assessment of a mobile gene pool in marine sediments. Environ Microbiol 10: 1024-1038.

Koenig JE, Bourne DG, Curtis B, Dlutek M, Stokes HW, Doolittle WF et al. (2011). Coral-mucus-associated Vibrio integrons in the Great Barrier Reef: genomic hotspots for environmental adaptation. ISME $J \mathbf{5}$ : 962-972.

Kümmerer K. (2001). Drugs in the environment: emission of drugs, diagnostic aids and disinfectants into wastewater by hospitals in relation to other sources-a review. Chemosphere 45: 957-969.

Lapara TM, Burch TR, McNamara PJ, Tan DT, Yan M, Eichmiller JJ. (2011). Tertiary-treated municipal wastewater is a significant point source of antibiotic resistance genes into Duluth-Superior harbor. Environ Sci Technol 45: 9543-9549.

Larouche A, Roy PH. (2011). Effect of attC structure on cassette excision by integron integrases. Mob DNA 2: 3 .

LeRoux F, Zouine M, Chakroun N, Binesse J, Saulnier D, Bouchier C et al. (2009). Genome sequence of Vibrio splendidus: an abundant planctonic marine species with a large genotypic diversity. Environ Microbiol 11: 1959-1970.

Levesque C, Piche L, Larose C, Roy PH. (1995). PCR mapping of integrons reveals several novel combinations of resistance genes. Antimicrob Agents Chemother 39: 185-191.

Łuczkiewicz A, Jankowska K, Fudala-Książek S, Olańczuk-Neyman K. (2010). Antimicrobial resistance of fecal indicators in municipal wastewater treatment plant. Water Res 44: 5089-5097.

Martínez JL. (2009). Environmental pollution by antibiotics and by antibiotic resistance determinants. Environ Pollut 157: 2893-2902.

Mazel D. (2006). Integrons: agents of bacterial evolution. Nat Rev Microbiol 4: 608-620.

Moura A, Henriques I, Smalla K, Correia A. (2010). Wastewater bacterial communities bring together broad-host range plasmids, integrons and a wide diversity of uncharacterized gene cassettes. Res Microbiol 161: 58-66.

Novo A, Manaia C. (2010). Factors influencing antibiotic resistance burden in municipal wastewater treatment plants. Appl Microbiol Biotechnol 87: 1157-1166.

Partridge SR, Tsafnat G, Coiera E, Iredell JR. (2009). Gene cassettes and cassette arrays in mobile resistance integrons. FEMS Microbiol Rev 33: 757-784.

Poirel L, Brinas L, Verlinde A, Ide L, Nordmann P. (2005). BEL-1, a novel clavulanic acid-inhibited extendedspectrum $\beta$-lactamase, and the class 1 integron In120 in Pseudomonas aeruginosa. Antimicrob Agents Chemother 49: 3743-3748.

Poirel L, Weldhagen GF, Naas T, De Champs C, Dove MG, Nordmann P. (2001). GES-2, a class A $\beta$-lactamase from Pseudomonas aeruginosa with increased hydrolysis of imipenem. Antimicrob Agents Chemother 45: 2598-2603.

Rizzo L, Manaia C, Merlin C, Schwartz T, Dagot C, Ploy MC et al. (2013). Urban wastewater treatment plants as hotspots for antibiotic resistant bacteria and genes spread into the environment: a review. Sci Total Environ 447C: 345-360.

Rodríguez-Rojas A, Rodríguez-Beltrán J, Couce A, Blázquez J. (2013). Antibiotics and antibiotic resistance: a bitter fight against evolution. Int J Med Microbiol 303: 293-297.

Rosewarne CP, Pettigrove V, Stokes HW, Parsons YM. (2010). Class 1 integrons in benthic bacterial communities: abundance, association with Tn402-like transposition modules and evidence for coselection with heavy-metal resistance. FEMS Microbiol Ecol 72: $35-46$.

Rowe-Magnus DA, Guerout A-M, Mazel D. (2002). Bacterial resistance evolution by recruitment of super-integron gene cassettes. Mol Microbiol 43: 1657-1669.

Stalder T, Alrhmoun M, Louvet J-N, Casellas M, Maftah C, Carrion C et al. (2013). Dynamic assessment of the floc morphology, bacterial diversity, and integron content of an activated sludge reactor processing hospital effluent. Environ Sci Technol 47: 7909-7917.

Stalder T, Barraud O, Casellas M, Dagot C, Ploy MC. (2012). Integron involvement in environmental spread of antibiotic resistance. Front Microbiol 3: 119.

Stokes HW, Gillings MR. (2011). Gene flow, mobile genetic elements and the recruitment of antibiotic resistance genes into Gram-negative pathogens. FEMS Microbiol Rev 35: 790-819.

Stokes HW, Hall RM. (1989). A novel family of potentially mobile DNA elements encoding site-specific geneintegration functions: integrons. Mol Microbiol 3: 1669-1683.

Szczepanowski R, Linke B, Krahn I, Gartemann KH, Gützkow T, Eichler W et al. (2009). Detection of 140 clinically relevant antibiotic-resistance genes in the plasmid metagenome of wastewater treatment plant bacteria showing reduced susceptibility to selected antibiotics. Microbiology 155: 2306-2319.

Uyaguari MI, Fichot EB, Scott GI, Norman RS. (2011). Characterization and quantitation of a novel $\beta$-lactamase gene found in a wastewater treatment facility and the surrounding coastal ecosystem. Appl Environ Microbiol 77: 8226-8233.

Wellington EMH, Boxall ABA, Cross P, Feil EJ, Gaze WH, Hawkey PM et al. (2013). The role of the natural environment in the emergence of antibiotic resistance in Gram-negative bacteria. Lancet Infect Dis 13: 155-165.

Wright MS, Baker-Austin C, Lindell AH, Stepanauskas R, Stokes HW, McArthur JV. (2008). Influence of in dustrial contamination on mobile genetic elements: class 1 integron abundance and gene cassette structure in aquatic bacterial communities. ISME J 2: 417-428.

Zhang X, Wu B, Zhang Y, Zhang T, Yang L, Fang HHP et al. (2009a). Class 1 integronase gene and tetracycline resistance genes tetA and tetC in different water environments of Jiangsu Province, China. Ecotoxicology 18: 652-660.

Zhang XX, Zhang T, Zhang M, Fang HHP, Cheng SP. (2009b). Characterization and quantification of class 1 integrons and associated gene cassettes in sewage treatment plants. Appl Microbiol Biotechnol 82: 1169-1177.

Supplementary Information accompanies this paper on The ISME Journal website (http://www.nature.com/ismej) 